$* * * * * * * * * * * * * * * * * * * * * * * * * * * * * * *$

Draft version, 01/12/21 This paper has not been peer reviewed. Please do not copy or cite without author's permission

$* * * * * * * * * * * * * * * * * * * * * * * * * * * * * * *$

Conversational Pragmatics: Memory Reporting Strategies in Different Social Contexts

Beatriz Martín-Luengo ${ }^{1}$, Karlos Luna ${ }^{2}$ \& Yury Shtyrov ${ }^{1,3}$

${ }^{1}$ Beatriz Martín-Luengo (corresponding author), Centre for Cognition and Decision Making, Institute for Cognitive Neuroscience, National Research University - Higher School of Economics, Moscow, Russian Federation

${ }^{2}$ Department of Psychology, Universidad Nacional de Colombia, Bogotá, Colombia

${ }^{3}$ Center of Functionally Integrative Neuroscience (CFIN), Department of Clinical

Medicine, Aarhús University, Aarhus, Denmark

Beatriz Martín-Luengo (corresponding author), Krivokolenniy sidewalk 3, entrance 2, 101000, Moscow, Russian Federation. Email: bmartinluengo@hse.ru

ORCID: (Dhttps://orcid.org/0000-0003-3642-5337

The authors have no conflicts of interest to disclose. 


\begin{abstract}
Conversational pragmatics studies, among others, factors that affect the information we share with others. Previous research showed that when participants are unsure about the correctness of an answer, they report fewer answers. This behavior strongly depends on the incentive structure of the social context where the question-response exchange takes place. In this research we studied how the different incentive structure of several types of social contexts affects conversational pragmatics and the amount of information we are willing to share. In addition, we also studied how different levels of knowledge may affect memory reporting in different social contexts. Participants answered easy, intermediate, and difficult general knowledge questions and decided whether they would report or withhold their selected answer in different social contexts: formal vs. informal, and constrained (a context that promotes providing only responses we are certain about) vs. loose (with an incentive structure that maximizes providing any type of answer). Overall, our results confirmed that social contexts are associated with a different incentive structure which affect memory reporting strategies, and that the effect of social contexts depended on the difficulty of the questions. Our results highlight the relevance of studying the different incentive structures of social contexts to understand the underlying processes of conversational pragmatics, and stress the importance of considering metamemory theories of memory reporting.
\end{abstract}

Keywords: Conversational pragmatics, memory reporting, confidence, social contexts 


\section{Conversational pragmatics: memory reporting strategies in different social contexts}

Efficient communication and exchange of information are essential for ensuring our survival in a dynamic environment. Yet, there are many points in research in human communication and, more specifically, in conversational pragmatics that require further investigation (Martín-Luengo et al., 2018). Most of the research on conversational pragmatics has been conducted from the point of view of what the listener would understand from the speaker's intended meaning (Cruz, 2014; De Neys, \& Schaeken, 2007; Dieussaert, et al., 2002; Mazzone, 2013; Papafragou \& Musolino, 2003). However, this is only one side of the coin in communication. To gain a more comprehensive understanding of communication process in context, we also need to understand it from the communicator's side.

In the present study, we report an experiment in which we studied the effect of the incentive structures subjacent to social situations in communication. To do so, we manipulated the type of social context where the communicational exchange takes place and the level of difficulty the speakers experience in providing the information in the message. We first review studies about how we regulate the amount of information we provide in the absence of a particular social context by relying on current metamemory theories of memory reporting. We then review studies on conversational pragmatics focusing on the regulation of informativeness in different social contexts. After that, we briefly review how the difficulty of finding the to-be-retrieved information may influence the information reported in such studies and, finally, we present the current study and our hypotheses.

\section{Tailoring a Message: The Report Option}


People do not speak in the same way and use the same language if they are talking to a close friend, a work colleague, or a stranger. Research in conversational pragmatics from the speakers' side confirm this observation: participants tailor the information they provide depending on the social context (Gibbs \& Bryant, 2008; McCallun et al., 2016; Martín-Luengo et al., 2018; Van der Hernst et al., 2002; Vandierendonck \& Van Damme, 1988). For example, participants provide different amount of details when they retell a previously presented story depending on the key audience they were approaching (as, e.g., shown by Vandierendock \& Van Damme, 1988, who used peers, public contest or a "Martians", as an audience).

One simple and efficient way to tailor a message is to decide whether to report a given piece of information or withhold it, effectively modifying the message depending on the circumstances. This decision, termed the report option (Koriat \& Goldsmith, 1996), is grounded in the metamemory processes of monitoring and control and is made based on a number of factors. The most relevant are (1) the confidence that the answer is correct and (2) the informativeness of the resulting answer (Goldsmith, 2016). In a conversational exchange, a communicator internally produces a piece of information, which is evaluated for its likelihood of being correct (confidence) and usefulness (informativeness). Subjective confidence and informativeness are then compared against a previously self-defined minimum criteria for a given situation and, if they are satisfied, then the answer is reported. Otherwise, it is withheld.1

1

If the criteria are not met, then the information can also be modified and a new comparison against criteria is made. There are several modifications that can be made depending on the nature of the information (see for example, Luna and Martín-Luengo, 2017; see also Goldsmith, 2016, for a review), but here we will focus in the report option and the decision to report or withhold an answer. 
Previous research has shown a trade-off between accuracy and informativeness in the information people provide (Ackerman, \& Goldsmith, 2008). One way to control this trade-off is the report option: in order to provide highly accurate information people might need to withhold some of the information, but when people want to maximize informativeness, they would report more information even though it may increase the chances of providing an incorrect answer (Koriat \& Goldsmith, 1996). Several factors affect the opposing tendencies to focus on informativeness and provide more elaborate answers vs, focussing on accuracy and reporting less elaborate/fewer answers. One of these factors is the social context.

\section{Social Context and their Incentives Structure}

The aforementioned informativeness and confidence criteria are affected by communication norms and pragmatic considerations relevant to a given context (Goldsmith, 2016). For example, Martín-Luengo et al. (2018, 2021) manipulated context formality and studied the tendency to report or withhold answers in formal and informal contexts. A context is formal when there are explicit rules that govern the situation, for example a job interview or a court testimony. A context is informal when such rules do not exist or are more flexible, for example when chatting with friends or being at a party. Past research has found that in formal settings participants are careful in balancing the information they report or withhold in order to increase the chances that they report correct information only, whereas in informal settings participants tend to report almost all their possible answers (Martín-Luengo et al., 2018; Martín-Luengo et al., 2021).

However, the metacognitive literature proposes that it is not the presence or absence of explicit rules as such that directly affect communication but rather the 
incentive structure of a given context that prompts different reporting strategies (Goldsmith, 2016). The incentive structure of a context refers to the perceived penalties and rewards for providing accurate answers on the one hand, or for providing informative answers on the other one. For example, in some contexts it may be more important to provide accurate information, that is, the situation rewards accuracy (e.g., successfully passing an exam), and in another contexts it rewards providing rich information (such as being accepted as a socially apt person at an informal event). Thus, it is not the context per se which prompts different reporting strategies but the association of the context with an underlying incentive structure which favours accuracy or informativeness. Depending on that incentive structure, people will apply a different reporting strategy. Also, it is important to mention that the perceived incentive structure of a given situation may vary from person to person and even within the same person between different moments of time. In this research, as well as in the research in which it is grounded, we studied people's perceptions and assumptions about the expected incentives in different social contexts, and not the real incentives of these situations.

However, it does not seem very reasonable to consider that any formal or informal situation is associated with a specific incentive structure and thus would promote the same report pattern. In this research, we propose that the incentive structure may also vary within different formal and informal contexts. For example, some contexts are characterized by the social pressure to present ourselves in the best possible light and make the best impression regarding our skills or knowledge, such as at a job interview (a formal context). In these contexts, people may tend to report information only when they have high confidence in its accuracy. In other words, people's report decisions may be 
highly constrained by confidence; thus, we termed them "constrained" contexts. The incentive structure of constrained contexts would favour accuracy. In some other settings, there may be a social demand to provide information and a tendency to report everything, or to adopt a more liberal criteria. For example, when testifying in a trial (also a formal context) eyewitnesses are urged to collaborate by giving all the information they might know about a criminal event (McCallun et al., 2016); while they may still refrain from answering or provide an "I don't know" answer, most of the times eyewitness provide any information they might think they know. In this type of context, the constraint of confidence can be lower, and thus the confidence in response correctness relevance only has a secondary role2. In these contexts, the answering strategy is better described as "loose" and the incentive structure would favour informativeness, even though the overall context is still very formal, similar to the previous example.

A parallel case can be made for informal contexts. A "first date" situation is an example of an informal context likely prompting a constrained answering strategy, because there are no explicit rules and people probably want to only share what they are confident in. Alternatively, an informal context with a loose answering strategy may be chatting with friends. Based on these definitions, it is possible to draw some similarities between the incentive structure at a job interview and at a date despite one context being formal and the other one - informal. In both cases, people might not report a lot of information unless they are reasonably certain about its correctness, that is, they favour

2

In support of this, Paulo, Albuquerque, and Bull (2016) showed that during a police interview witnesses produced statements accompanied by markers of uncertainty (e.g., I believe, maybe), showing that they were willing to report information event though they had low confidence in it. Thus, reporting information (i.e., the informativeness of the answer) is perceived as more relevant than the accuracy of the retrieved memory. 
accuracy. Likewise, there are similarities between the incentives when testifying in a trial and chatting with friends. In both loose contexts the incentive would be to report maximum information, that is, they both favour informativeness. To verify this suggestion, the present research tested the different patterns of reporting derived from the different incentive structure of each context.

\section{Difficulty of the Questions}

Another issue, which has been treated differently in studies on conversational pragmatics, is the difficulty of the material. In some studies, participants had access to the information during the main task (Gibbs \& Bryant, 2008; Van der Hernst et al., 2002) or the information was presented few minutes beforehand (Vandierendonck \& Van Damme, 1988), which made the tasks relatively easy. In other studies, difficult general-knowledge questions were used under the assumption that answers to easy questions would be reported regardless of the context (Martín-Luengo et al., 2018, 2021). This idea is consistent with the criteria to report a piece of information. With easy questions, confidence would probably be high enough to satisfy the criterion, and thus participants will not have to withhold information. However, that idea was never empirically tested. In this research, therefore, our secondary objective was to examine the effect of incentives in the willingness to report or withhold information for questions varying in difficulty.

\section{The Present Research}

In summary, we studied the effects of the incentive structure prompted by different social contexts. We manipulated both the formality of the context (formal, informal) and the answering strategy the context elicits (loose, constrained) in 
communicating individual responses to easy, intermediate, and difficult questions. We particularly focused on the decision to report or withhold information in a conversational exchange as a window onto the information the speaker wants to provide. In line with our previous research (Martín-Luengo et al., 2018, 2021), we expected that informal contexts would be associated with an incentive favouring informativeness and thus expected participants to report more information in an informal than in a formal context. However, in light of the discussion of potential differences in the accuracy-informativeness incentive structure that are not tied to formality or informality per se, we also expected that participants would exhibit a higher tendency to report their answers in "loose" contexts (prioritizing informativeness) than in "constrained" contexts (prioritizing accuracy), regardless of the formality of the social context. Finally, we ask whether the item difficulty may enter into interaction with the incentive structure of the contexts. If so, this influence might be seen for some levels of difficulty such as intermediate and difficult since there should not be much need of regulation with the easy questions. At this stage, this hypotheses is entirely speculative, particularly as Koriat \& Goldsmith (1996)'s metacognitive model does not predict such influence as long as the reporting rate is adjusted in mean confidence.

To test these hypotheses, we conducted an experiment in which participants answered general knowledge questions and rated the confidence in their answers prior to being informed of the specific context. After that, one of the four possible social contexts was introduced and participants had decide whether to report or withhold their answer in that specific context. We used a large amount of general-knowledge questions validated previously in a large sample (Martín-Luengo et al., 2020) to ensure that each context had 
a similar amount of easy, intermediate, and difficult questions in a counterbalanced fashion.

\section{Method}

\section{Participants}

Twenty-five participants ( 11 females, mean age $=21.03, S D=3.92)$ recruited on social media took part in the experiment for a small monetary compensation.

\section{Materials and Design}

Four hundred and two multiple-choice general-knowledge questions (GKQ) were used in the experiment. Four hundred questions were used in the main experimental part and two for practice. The questions were selected from Martín-Luengo et al. (2020). The GKQ covered different topics - history, chemistry, biology, literature, spelling and punctuation, and geography. The GKQ were selected to include all levels of difficulty: easy, intermediate, and difficult. For each question, participants selected one out of four alternatives and rated how confident they were in their answers on a scale from $0 \%$ (totally unsure) to $100 \%$ (totally sure), graded in $10 \%$ steps. The design was a 2 Formality (formal, informal) X 2 Answering Strategy (constrained, loose) x 3 Question Difficulty (easy, intermediate, difficult), with the three variables manipulated within participants in a fully counterbalanced fashion. Main measures were accuracy, confidence in the correctness of the selected answer, and proportion of reported answers.

\section{Procedure}

The experiment was programmed using Experiment Builder 2.3.38 (SR Research, Toronto, Canada), and consisted of one practice session with two questions and eight 
experimental blocks with 50 questions each. The order of the questions in the experimental blocks, the placement of the alternatives on the screen, and the social context for each question were fully counterbalanced. No more than two questions were placed in the same context in a row. All participants had the same practice questions, which were not used in the main session.

Figure 1 shows an overview of the steps followed on a given trial. Table 1 shows the descriptions of the pictures for each context that appears in Figure 1.Participants were tested individually on a computer. First, they read and signed the informed consent form and provided demographic data. Then, they read the instructions and completed two training questions before beginning the experimental phase. A question appeared on the screen for four seconds and the participants were instructed that they had three seconds to provide the answer. They were shown four alternative answers and had to use the mouse to click on the correct one. The participants then rated their confidence that their answer was correct. Finally, participants were shown a specific context which consisted of a picture with a brief description (see Table 1) and chose whether they preferred to report or withhold their answer in that specific context. The experiment lasted approximately 2.5 hours. To prevent fatigue, participants were also given the chance to take a brief rest after each question. There were also breaks of 2-3 minutes between blocks in which participants had the chance to leave the room, stretch their muscles, eat or drink something, and use the toilet if needed.

\section{Results}

Unless otherwise mentioned, we report 2 (formality: formal, informal) x 2 (answering strategy: constrained, loose) x 3 (question difficulty: easy, intermediate, 
difficult) ANOVA, and pairwise comparisons with the Student's $t$ test and Bonferroni correction for multiple comparisons when appropriate. We also report partial-eta squared $\left(\eta \mathrm{p}^{2}\right)$, eta-squared $\left(\eta^{2}\right)$, and Cohen's $d_{a v}$ as measures of effect size. Question difficulty was manipulated a posteriori. Each participant answered 100 questions for each context. To ensure a similar amount of questions per difficulty level, we chose the cut-off points of .40 and .70. These limits divided the amount of questions in each of the contexts in three levels with approximately one third of the questions on each one.

Our main hypotheses pertained to the proportion of reported answers. However, differences in accuracy and confidence could affect the decision to report or withhold an answer. Thus, we first conducted analyses to check whether accuracy and confidence were similar. A full report of these analyses is presented in the Supplemental Materials but we included the main descriptive statistics in Table 2 for completeness. We also provide here a brief summary of these results. For accuracy, we found no differences per formality or answering strategy, and found the expected increase in accuracy as questions got easier. As per confidence, the same results were obtained but we also found a significant interaction between formality, answer strategy, and difficulty. Further analyses showed differences in confidence only for easy questions, and that metamemory measures such as calibration and resolution were not affected. However, as confidence highly affects the decision to report an answer (Koriat \& Goldsmith, 1996), this constitutes a strong theoretical reason to include it as a covariate to avoid any confounds based on differences in confidence per se; we therefore ran an ANCOVA using confidence as covariate. This adjusted the reporting rate and eliminated any effect that was a consequence of prior differences in confidence. For example, the ANCOVA below 
equated confidence for the three levels of items difficulty. If question difficulty had an effect beyond confidence, then the ANCOVA should show differences in that variable. Having confidence controlled and equated in all conditions, the measure in the adjusted reporting rate analysis is better interpreted as showing whether participants were conservative or liberal with respect to their own confidence level.

\section{Adjusted Reporting Rates}

We conducted an analysis of covariance (ANCOVA) with the three variables, formality, answering strategy, and difficulty, using the $e z$ package (Lawrence, 2016) in R (R Core Team, 2020). We entered the proportion of reported answer as measure and confidence as covariate. Higher adjusted rates are interpreted as a conservative reporting policy (i.e., a focus in accuracy) and lower adjusted rates are interpreted as a liberal policy (i.e., a focus in informativeness).

The $3 \times 2 \times 2$ ANCOVA showed a more liberal reporting strategy in the informal ( $M$ $=.70, S D=.15)$ than in the formal contexts $(M=.68, S D=.16), F(1,24)=19.84, p<$ $.001, \eta p^{2}=.45$, and no differences by answering strategy, $F(1,24)=2.06, p=.164, \eta p^{2}=$ .08 , or by question difficulty, $F(2,48)=0.20, p=.822, \eta^{2} \mathrm{p}=.01$. This latter result indicated that any effect of question difficulty in actual reporting rates could in fact be due to differences in confidence. Descriptive statistics of proportions adjusted by the covariate and unadjusted are presented in Table 2 . The interactions formality x difficulty, $F(2,48)=3.42, p=.041, \eta p^{2}=.12$, and answering strategy $x$ difficulty, $F(2,48)=3.98, p$

$.025, \mathrm{np}^{2}=.14$, were significant.

Pairwise comparisons were conducted with the emmeans package (Lenth, 2019) in R (R Core Team, 2020) to ensure that comparisons were made over the response 
means adjusted by the confidence covariate and not over the raw, unadjusted, means. As our main interest was the reporting strategy used depending on the social context we focused on pairwise comparisons within each difficulty level. For each interaction, six pairwise comparisons were conducted. We applied Bonferroni correction and alpha level was set at .008. For difficult questions, participants applied a more liberal reporting policy in the informal $(M=.70, S D=.16)$ than in the formal context $(M=.67, S D=.15)$, $t(71.9)=3.03, p=.003, d_{a v}=0.18$, and also in the loose $(M=.71, S D=.15)$ than in the constrained context $(M=.67, S D=.16), t(71)=2.93, p=.004, d_{a v}=0.21$. For the intermediate questions, participants also applied a more liberal policy in the informal ( $M$ $=.70, S D=.16)$ than in the formal context $(M=.67, S D=.17), t(71.9)=4.09, p<.001$, $d_{a v}=0.23$, and there were no differences for easy questions.

\section{$P_{r c}$ (Report-criterion probability) Analyses3}

The above results showed that the incentives structure of social contexts only affected reporting strategy for intermediate and difficult items. To further test that conclusion, we conducted another analysis based on the confidence threshold participants used to decide whether a response should be reported or withheld. That threshold can be computed using a procedure introduced by Koriat and Goldsmith (1996; for further explanation on computation, see also Goldsmith and Koriat, 2007). The threshold is usually referred to as the report-criterion probability, or $P_{r c}$. As in the previous analyses, a higher $P_{r c}$ means that fewer answers are reported and a more conservative strategy is employed, that is, the focus is on accuracy; the opposite is true for lower $P_{r c}$. We computed $P_{r c}$ for each participant on each of the four social contexts and then examined

3

We thank Morris Goldsmith for suggesting this analysis. 
the confidence distributions for the easy, intermediate, and difficult items to identify the proportion of items at each difficulty level that fall in the range between the highest and lowest $P_{r c}$ value for the four contexts. When the effect of social context is stronger for a given difficulty level, then there should be more responses falling between the highest and lowest $P_{r c}$.

Following that logic, we computed the number of responses rated with confidence between the minimum and maximum $P_{r c}$ per participant and difficulty level and entered the results into a one-way ANOVA 3 (using difficulty as a factor). The results showed significant differences, $F(2,48)=24.72, p<.001, \eta^{2}=.51$. Pairwise comparisons showed that the number of responses between the minimum and maximum $P_{r c}$ was higher for the difficult $(M=23.32, S D=12.03, C I 18.6,28.04])$ and intermediate $(M=25.08$, $S D=11.83, C I[20.44,29.72])$ questions than for the easy ones $(M=13.60, S D=8.01$, $C I[10.46,16.74]), t(24)=4.49, p<.001, d_{a v}=0.95$ and $t(24)=6.34, p<.001, d_{a v}=1.14$, respectively, without differences between difficult and intermediate questions, $t(24)=$ $1.54, p=.137, d_{a v}=-0.15$. In sum, this analysis confirmed that the incentives structure of social contexts has a stronger effect in reporting strategies when answering intermediate and difficult questions than when answering easy questions.

\section{Discussion}

In this study, we examined context-dependent conversational pragmatics from the perspective of the speaker. In particular, we investigated the effect of the incentive structure subjacent to different social contexts and levels of difficulty in the amount of information the speakers share. Participants answered general-knowledge questions 
covering all levels of difficulty (easy, intermediate, difficult) and decided whether to report or withhold their answers in a particular social context. The main results showed that the incentive structure of social contexts categorized as formal or informal or as constrained or loose affected reporting rates and, thus, the amount of information speakers shared in these contexts. This is relevant for studying conversational pragmatics because it shows that social context influences reporting strategies, thus confirming previous suggestions (Ackerman \& Goldsmith, 2008). The results also show that the influence of social context vanishes as the certainty regarding the answers' correctness increases. In general, the results are highly consistent with the framework of the strategic regulation of memory reporting by Koriat and Goldsmith (1996), as extended by Goldsmith (2016): contextual factors and communication norms stemming from different contexts alter participants' perception of the rewards and penalties involved in reporting more or less information and, as a consequence, affect the amount of information they report.

This research expands and better characterizes the influence of social contexts on memory reporting. Previous research manipulated only one context dimension, formal vs. informal (Martín-Luengo et al., 2018, 2021), but here we altered the structure of incentives by considering the general answering strategy expected in a given context. Our research showed that binary categories such as formality may not be the best way to study the effect of social context, because the possible scenarios within that category (e.g., formal or informal) may not be linked to the same incentives structure and elicit the same response criterion. The focus on the incentives structure of different social contexts allowed us to further investigate other nuances of formal and informal contexts. The 
results highlight the significant role of incentives for reporting depending on the social context and the variety that any broad category of context can include.

This research also showed that the strategic regulation of the informativenessaccuracy trade-off can be investigated with general-knowledge questions of all difficulties. Previous research in conversational pragmatics and memory reporting was conducted with difficult questions (Martín-Luengo et al., 2018, 2021), following the logic that only when the questions are difficult there is a need to regulate the amount of information provided through reporting or withholding answers. Interestingly, the results here suggested that questions of intermediate difficulty are also subject to regulation of accuracy and social context effects. Participants did not report all the answers for easy questions but, in this case, the social context did not affect the reporting. These results open interesting venues for future research, such as the factors that affect the regulation of accuracy for questions of different difficulties. Our research showed that social context affected the responses to intermediate and difficult questions but not to easy ones. This does not exclude the possibility that other, yet unknown, factors may affect easy questions, which remains to be addressed in future studies.

Considering the results based on the proportion of reported answers, we found that the context did not affect reporting for easy questions, while for intermediate questions it was only influenced by formality, and for difficult questions both types of context influenced reporting. The results of the report criterion were consistent, but less nuanced in that they do not identify the specific context affecting responses. These results show that the effect of social context on memory reporting is more complex than previously suggested. One tentative and speculative explanation for this might be that 
when questions are intermediate or difficult, threats to our self-image and the way people present themselves may be more evident, which, in turn, could increase the perceived strength of the rewards and penalties for focusing on accuracy or informativeness in a given situation. However, for easy questions participants are more prone to ignore the context, maybe because the chances of a mistake are low and the perceived consequences to self-image are also minimal.

We expected that social context may not affect reporting strategies for easy questions because most of the answers would be rated with high confidence and, thus, reported. However, a similar argument could be made for answers to difficult questions. If they are rated with low confidence, then they should be mostly withheld. From this perspective, only the condition with intermediate questions should be sensitive enough to show the effect of social context. However, this idea was not supported because the results showed an effect of context for difficult questions. One explanation for this is that, as mentioned, the perceived incentives and penalties for focusing on accuracy or informativeness may be stronger when questions are difficult. Another explanation is that difficult questions were not difficult enough. Arguing for the contrary, accuracy for difficult questions was around .25, which is the baseline in a four-alternative test. However, confidence for difficult questions was near 50\%, which suggests that participants did not perceive questions as that much difficult. If participants considered difficult questions as intermediate, that could explain the effect of social context for difficult questions. Future research should try to test both of these alternatives to further advance our understanding of the effect of perceived incentives of social situations on memory reporting strategies. 
Finally, another novel aspect of the present research is the use of multiple-choice questions instead of cued-recall or free recall questions. In previous studies, cued-recall questions were used as a proxy to what a conversation is, that is, a sequence of comments or questions and answers. This notwithstanding, it is also the case that when we are involved in a conversation, sometimes we also provide options along with our questions. Recognition tests are known to be easier than cued-recall tests because they are affected by familiarity (Martín-Luengo et al., 2012; Richardson-Klavehn, \& Bjork, 1988; Tulving, 1985), and at the same time they provide a straightforward way for experimental manipulations of individual items and easy quantification of results. This shows that the effect of social context on memory reporting goes beyond the nature of the answers.

\section{Limitations}

While clearly demonstrating novel findings, this study also has some limitations. First, the computerized administration of the questions was not particularly representative of how a natural conversation or interview unfolds. Previous research used different methods to try to mimic conversational exchanges. For example, Smith and Clark (1993) presented general knowledge questions orally (for a similar procedure, see Kim, 2013). In the present case, due to the manipulation of the social contexts we decided against an oral experiment because it would have likely been more difficult for participants to imagine themselves in different social contexts represented by the very same experimenter than identifying the different social contexts using a graphical image. Second, context changed from one question to the next one in random order. While a block design with one block for each social context could have been more ecological, we decided to change contexts pseudo-randomly as a way to test the strength of the implications of each context: a block 
design could have allowed participants to set a priory a strategy on each context-block thus decreasing the variability of the results. Future research should strive for complying a more naturalistic experimental setting without compromising the quality of the results.

In line with these limitations, a more naturalistic procedure could have allowed to collect other conversational markers such as silences, hesitations, humbling, etc. These markers convey a large amount of valuable information that may help further understand participant's conversational behaviour. Future research on memory reporting would be greatly improved by the inclusion of these conversational markers in the context of a more naturalistic conversation.

Another limitation of this study is that each individual may react and behave in a particular social context in a rather varied way. Reactions can vary even depending on the emotional, psychological, or cognitive state of the same person. The present research was not intended to study individual differences and that is why we framed the social contexts with the most prototypical and representative situations. Participants’ responses to prototypical situations inform us about what they believe is the incentive structure in these situations and what they think is the most appropriate reporting strategy. Thus, the results presented in this research are informative about general patterns of memory reporting, the perceived expectations in a given social context, and what the usual behaviour in response to social challenges is, and should not be interpreted as indicative of individual responses in particular situations.

\section{Concluding Remarks}

This study showed the importance of the incentives structure of a given social context to decide on the best reporting strategy, the one focused on accuracy and 
reporting fewer answers or the one focused on informativeness and hence reporting more answers. In addition, this research also showed that the difficulty or materials is relevant to understand reporting strategies, with easy questions being seemingly less sensitive to variations in social context. This helps advance our understanding of how social situations shape and affect communication flow, in particular how much information people are willing to share. Future research should study other ways people can use to modify their discourse behavior, to further understand human communication.

\section{Acknowledgements}

This study was funded by the Russian Science Foundation (project code RSFU 19-18-00534) and conducted in the behavioural laboratories of the Center for Cognition and Decision Making, Institute for Cognitive Neuroscience, NRU-HSE. 


\section{References}

Ackerman, R., \& Goldsmith, M. (2008). Control over grain size in memory reporting With and without satisficing knowledge. Journal of Experimental Psychology: Learning, Memory, and Cognition, 34, 1224-1245. https://doi.org/10.1037/a0012938

Cruz, M. P. (2014). Pragmatic failure, epistemic injustice and epistemic vigilance. Language \& Communication, 39, 34-50. https://doi.org/10.1016/j.langcom.2014.08.002

De Neys, W., \& Schaeken, W. (2007). When people are more logical under cognitive load: Dual task impact on scalar implicature. Experimental Psychology, 54, 128133. https://doi.org/10.1027/1618-3169.54.2.128

Dieussaert, K., Schaeken, W., \& d'Ydewalle, G. (2002). The relative contribution of content and context factors on the interpretation of conditionals. Experimental Psychology, 49, 181-195. https://doi.org/10.1026//1618-3169.49.3.181 .

Griffin, D., \& Tversky, A. (1992). The weighing of evidence and the determinants of confidence. Cognitive Psychology, 24, 411-435. https://doi.org/10.1016/00100285(92)90013-R

John, L. K., Barasz, K., \& Norton, M. I. (2016). Hiding personal information reveals the worst. Proceedings of the National Academy of Sciences, 113, 954-959. https://doi.org/10.1073/pnas.1516868113 
Kim, M. S. (2013). Answering questions about the unquestionable in Korean conversation. Journal of Pragmatics, 57, 138-157. https://doi.org/10.1016/j.pragma.2013.08.004

Koriat, A. (2008). Subjective confidence in one's answers: The consensuality principle. Journal of Experimental Psychology: Learning, Memory, and Cognition, 34, 945-959. https://doi.org/10.1037/0278-7393.34.4.945

Koriat, A., Goldsmith, M., \& Pansky, A. (2000). Toward a psychology of memory accuracy. Annual Review of Psychology, 51, 481-537. https://doi.org/10.1146/annurev.psych.51.1.481

Goldsmith, M. (2016). Metacognitive quality-control processes in memory retrieval and reporting. In J. Dunlosky \& S. K. Tauber (Eds.), The Oxford handbook of metamemory (pp. 357-385). Oxford University Press.

Goldsmith, G., \& Koriat, A. (2007). The strategic regulation of memory accuracy and informativeness. En A. B. Ross (Ed.), The Psychology of learning and motivation, Vol.48: Skill and strategy in memory use (pp. 1-60). Elsevier.

Lawrence, M. A. (2016). ez: Easy Analysis and Visualization of Factorial Experiments. R package version 4.4-0. https://CRAN.R-project.org/package=ez

Lenth, R. (2019). emmeans: Estimated Marginal Means, aka Least-Squares Means. R package version 1.4.3.01. https://CRAN.R-project.org/package=emmeans

Luna, K., \& Martín-Luengo, B. (2012). Confidence-accuracy calibration with general knowledge and eyewitness memory cued recall questions. Applied Cognitive Psychology, 26, 289-295. https://doi.org/10.1002/acp.1822 
Luna, K., \& Martín-Luengo, B. (2017). Using the regulation of accuracy to study performance when the correct answer is not known. Scandinavian Journal of Psychology, 58, 275-283. https://doi.org/10.1111/sjop.12369

Martín-Luengo, B., Luna, K., \& Migueles, M. (2012). Efecto del tipo de prueba de evaluación en la memoria y valoración de marcas publicitarias [Effect of the type of memory test on the evaluation of brands]. Escritos de Psicología/Psychological Writings, 5, 24-30. https://doi.org/10.5231/psy.writ.2012.0611

Martín-Luengo, B., Nunez Vorobiova, A., Feurra, M., Myachykov, A., \& Shtyrov, Y. (2020). Social context-dependent role of the left medial prefrontal cortex in communicational exchanges: rTMS evidence. Manuscript submitted for publication.

Martín-Luengo, B., Shtyrov, Y., Luna, K., \& Myachykov, A. (2018). Different answers to different audiences: Effects of social context on the accuracy-informativeness trade-off. Memory, 26, 993-1007. https://doi.org/10.1080/09658211.2017.1420196

Martín-Luengo, B., Zinchenko, O., Alekseeva, M., \& Shtyrov, Y. (2020). Russian norms for 500 general-knowledge questions. Frontiers in Psychology, 11, 2269. https://doi.org/10.3389/fpsyg.2020.545304 .

Mazzone, M. (2013). Attention to the speaker. The conscious assessment of utterance interpretations in working memory. Language \& Communication, 33, 106-114. https://doi.org/10.1016/j.langcom.2013.01.001 
Paulo, R. M., Albuquerque, P. B., \& Bull, R. (2016). The enhanced cognitive interview: expressions of uncertainty, motivation and its relation with report accuracy. Psychology, Crime \& Law, 22, 366-381.

https://doi.org/10.1080/1068316X.2015.1109089

R Core Team (2020). R: A language and environment for statistical computing. R Foundation for Statistical Computing [Computer software]. https://www.rproject.org/.

Smith, V. L., \& Clark, H. H. (1993). On the course of answering questions. Journal of Memory and Language, 32, 25-38. https://doi.org/10.1006/jmla.1993.1002

Richardson-Klavehn, A. \& Bjork, R. A. (1988). Measures of memory. Annual Review of Psychology, 39, 475-543. https://doi.org/10.1146/annurev.ps.39.020188.002355.

Tulving, E. (1985). Memory and consciousness. Canadian Psychologist, 26, 1-12. https://doi.org/10.1037/h0080017. 


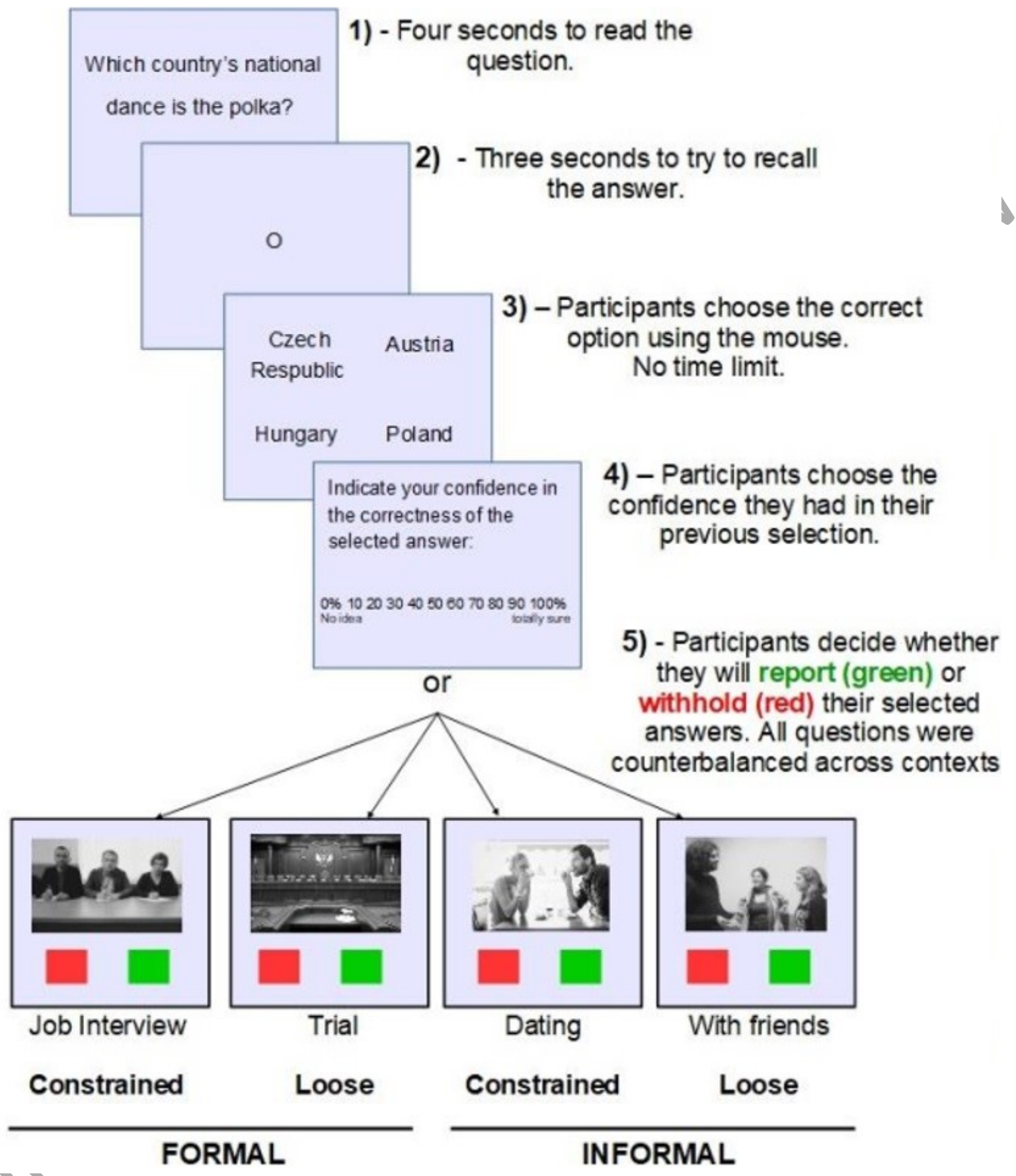

Figure 1. Experimental procedure for each trial. 
Table 1. Translation into English of the Descriptions for Each Condition.

\begin{tabular}{|l|l|l|}
\hline \multirow{2}{*}{$\begin{array}{l}\text { Formal } \\
\text { contexts }\end{array}$} & Constrained & $\begin{array}{l}\text { Job Interview: Imagine that you are in an important job } \\
\text { interview. You really need this job. You feel the tension, but } \\
\text { you still try to look like an expert in the field. }\end{array}$ \\
\cline { 2 - 3 } & Loose & $\begin{array}{l}\text { Court: Imagine that you are testifying in court and that the } \\
\text { judge is asking you questions. You do not know whether } \\
\text { your answers will be helpful, but they might help to convict } \\
\text { the killer. }\end{array}$ \\
\hline \multirow{2}{*}{$\begin{array}{l}\text { Informal } \\
\text { contexts }\end{array}$} & Constrained & $\begin{array}{l}\text { Dating: Imagine that you are hanging out with someone you } \\
\text { would like to date. You are having fun but you are still } \\
\text { trying to make a good impression. }\end{array}$ \\
\cline { 2 - 3 } & Loose & $\begin{array}{l}\text { Conversation with friends: Imagine that you are with } \\
\text { friends, having a good time. You feel relaxed and glad to be } \\
\text { with them. You are having a cheerful conversation about } \\
\text { different topics. }\end{array}$ \\
\hline
\end{tabular}


Table 2. Means (Standard Deviations) of the Main Measures per Context and Question Difficulty. The unadjusted values of the reporting rates have no direct interpretation but are included for completeness.

\begin{tabular}{|c|c|c|c|c|}
\hline & \multicolumn{2}{|c|}{ FORMAL } & \multicolumn{2}{|c|}{ INFORMAL } \\
\hline & Constrained & Loose & Constrained & \\
\hline \multicolumn{5}{|l|}{ Accuracy } \\
\hline Difficult & $.26(.07)$ & $.24(.11)$ & $.25(.07)$ & \\
\hline Intermediate & $.55(.14)$ & $.50(.14)$ & $.53(.14)$ & $.55(.16)$ \\
\hline Easy & $.84(.12)$ & $.86(.10)$ & & $.84(.12)$ \\
\hline \multicolumn{5}{|l|}{ Confidence } \\
\hline Difficult & $47.25(16.86)$ & $49.27(16.09)$ & $48.95(16.18)$ & $49.08(16.21)$ \\
\hline Intermediate & $55.54(15.34)$ & $54.70(15.98)$ & $53.97(15.76)$ & $56.33(16.23)$ \\
\hline Easy & $73.62(13.06)$ & $77.79(11.35)$ & $76.64(11.81)$ & $75.57(12.25)$ \\
\hline \multicolumn{5}{|c|}{ Proportion Reported (Adjusted by the Covariate) } \\
\hline Difficult & $.66(.16)$ & $.68(.16)$ & $.68(.16)$ & $.73(.16)$ \\
\hline Intermediate & $.65(.16)$ & $.68(.19)$ & $.71(.16)$ & $.69(.16)$ \\
\hline Easy & $.69(.17)$ & $.68(.16)$ & $.70(.14)$ & $.68(.17)$ \\
\hline \multicolumn{5}{|c|}{ Proportion Reported (Unadjusted by the Covariate-Raw Proportion) } \\
\hline Difficult & $55(.21)$ & $.59(.19)$ & $.58(.20)$ & $.63(.21)$ \\
\hline Intermediate & 19) & $.63(.22)$ & $.66(.20)$ & $.66(.19)$ \\
\hline Easy & $.81(.12)$ & $.85(.11)$ & $.86(.10)$ & $.82(.13)$ \\
\hline
\end{tabular}




\section{Supplemental Materials}

Conversational Pragmatics: Memory Reporting Strategies in Different Social

Contexts 
In the research reported in the main manuscript, we were interested in the reporting strategies depending of the incentive structure of different social contexts. In order to properly examine reporting strategies, we first needed to verify that the accuracy and the confidence levels were equally distributed among conditions. Thus, we report here analyses of accuracy and confidence per context and difficulty level.

\section{Accuracy}

See Table S1 for descriptive statistics. There were no differences for formality, $F(1,24)=0.07, p=.789, \eta_{\mathrm{p}}^{2}<.01$, or answering strategy, $F(1,24)=1.72, p=.202, \eta_{\mathrm{p}}{ }^{2}=$ .07 , and there was an expected effect of question difficulty, $F(2,48)=541.83, p<.001$, $\eta_{\mathrm{p}}{ }^{2}=.96$. No interactions were significant.

Table S1. Means (Standard Deviations) of Accuracy and Confidence per Context and Question Difficulty.

\begin{tabular}{|c|c|c|c|c|}
\hline & & & INFOF & RMAL \\
\hline & & Loose & Constrained & Loose \\
\hline ccuracy & & & & \\
\hline Difficult & $.26(.07)$ & $.24(.11)$ & $.25(.07)$ & $.22(.11)$ \\
\hline Intermediate & $.55(.14)$ & $.50(.14)$ & $.53(.14)$ & $.55(.16)$ \\
\hline Easy & $.84(.12)$ & $.86(.10)$ & $.85(.09)$ & $.84(.12)$ \\
\hline$e$ & & & & \\
\hline & 47.25 (16.86) & 49.27 (16.09) & 48.95 (16.18) & $49.08(16.21)$ \\
\hline Intermediate & $55.54(15.34)$ & $54.70(15.98)$ & $53.97(15.76)$ & 56.33 (16.23) \\
\hline Easy & 73.62 (13.06) & 77.79 (11.35) & 76.64 (11.81) & $75.57(12.25)$ \\
\hline
\end{tabular}

Note: data in this table are also presented in the main text. We repeat the information for the convenience of the reader. 


\section{Confidence}

See Table S1 for descriptive statistics. Confidence was rated after the answer was provided but before the context was presented. Thus, we expected an effect of difficulty, with higher confidence as question difficulty decreased, and no differences by context. An ANOVA with confidence in the answers showed no differences for formality, $F(1,24)$ $=0.40, p=.534, \eta_{\mathrm{p}}^{2}=.02$, differences for answering strategy, $F(1,24)=4.77, p=.039$, $\eta_{p}^{2}=.17$, and an effect of question difficulty, $F(2,48)=152.22, p<.001, \eta_{p}^{2}=.86$. There was also an interaction between the three variables that qualified the main effects, $F$ (2, 48) $=5.64, p=.006, \eta_{\mathrm{p}}^{2}=.19$. There were no other interactions.

Exploration of the interaction showed that differences between contexts were limited to the easy questions. Easy questions that were placed later in the formal-loose context were rated with higher confidence than those placed later in the formalconstrained context, $t(24)=4.09, p<.001, d=0.33$, with no other differences regarding contexts. This difference was unexpected because, as mentioned, contexts were presented after confidence was registered and we fully counterbalanced conditions.

To further test whether differences in confidence depending on the type of context affected metamemory, we also analysed confidence in relation with accuracy through calibration and resolution analyses (see Table S2 for the main descriptive statistics). To ensure enough data points in each condition, we collapsed the 11 confidence points into five. Confidence levels 0 and 100 are qualitatively different than others because they show that the participant was guessing or completely certain, while the intermediate levels show different degrees of uncertainty. Thus, we collapsed levels 10-20-30, 40-50- 
60, 70-80-90, and maintained levels 0 and 100.

Table S2. Means (Standard Deviations) of the Calibration Index and Gamma

\begin{tabular}{lcccc}
\hline & \multicolumn{2}{c}{ FORMAL } & \multicolumn{2}{c}{ INFORMAL } \\
\hline & Constrained & Loose & Constrained & Loose \\
\hline Calibration Index & & & & \\
$\quad$ Difficult & $0.18(0.10)$ & $0.20(0.10)$ & $0.18(0.12)$ & $0.21(0.12)$ \\
Intermediate & $0.11(0.07)$ & $0.10(0.07)$ & $0.09(0.06)$ & $0.11(0.05)$ \\
Easy & $0.07(0.06)$ & $0.07(0.05)$ & $0.06(0.06)$ & $0.07(0.06)$ \\
\hline Gamma Correlation & & & & \\
$\quad$ Difficult & $.12(.49)$ & $-.06(.47)$ & $.15(.50)$ & $-.03(.41)$ \\
Intermediate & $.30(.30)$ & $.29(.33)$ & $.40(.27)$ & $.41(.30)$ \\
Easy & $.63(.36)$ & $.62(.33)$ & $.58(.45)$ & $.60(.34)$ \\
\hline
\end{tabular}

Calibration measures the extent to which confidence matches accuracy. A participant would be perfectly calibrated when their answers rated with $50 \%$ confidence are 50\% accurate and so on. A calibration index (see Brewer et al., 2002 for the calculations) of 0 indicates a perfect calibration and higher values suggest worse calibrations. There were no differences in formality, $F(1,24)=0.22, p=.642, \eta_{\mathrm{p}}^{2}<.01$, or answering strategy, $F(1,24)=3.62, p=.069, \eta_{\mathrm{p}}{ }^{2}=.13$. Calibration was better as difficulty decreased, $F(1,24)=27.46, p<.001, \eta_{\mathrm{p}}^{2}=.53$, which is consistent with the idea that good knowledge is accompanied by good knowledge about knowing. No interaction was significant.

Similarly, differences in confidence did not affect resolution. Resolution measures the degree to which confidence can discriminate between correct and incorrect responses and is measured with gamma correlations. Gamma ranges from +1 , perfect positive 
discrimination (i.e., all correct answers are rated with the highest confidence), to -1, meaning perfect negative discrimination (i.e., all the correct responses are rated with the lowest confidence). A gamma equal to zero is interpreted as no discrimination. Gamma was computed for each individual and condition and pooled together. There were no differences in formality, $F(1,24)=0.56, p=.461, \eta_{p}^{2}=.023$, or answering strategy, $F(1$, $24)=2.72, p=.113, \eta_{\mathrm{p}}^{2}=.10$, and resolution improved as difficulty decreased, $F(1,24)=$ 39.93, $p<.001, \eta_{\mathrm{p}}^{2}=.62$. There were no interactions.

Taken together, the results indicate that the highest confidence in answers that were later in the formal-loose condition for easy questions did not affect other metamemory measures. However, reporting or withholding an answer is highly based on confidence and that result could explain differences in the proportion of reported answers. To control for this alternative explanation, in the analyses of the proportion of reported answers in the main text we included confidence as a covariate.

\section{References}

Brewer, N., Keast, A., \& Rishworth, A. (2002). The confidence-accuracy relationship in eyewitness identification: The effects of reflection and disconfirmation on correlation and calibration. Journal of Experimental Psychology: Applied, 8, 44- 